\title{
A critical overview of the imaging arm of the ASAS criteria for diagnosing axial spondyloarthritis: what the radiologist should know
}

\author{
Üstün Aydıngöz, Adalet Elçin Yıldız, Zeynep Maraş Özdemir, Seray Akçalar Yıldırım, Figen Erkuş, \\ Fatma Bilge Ergen
}

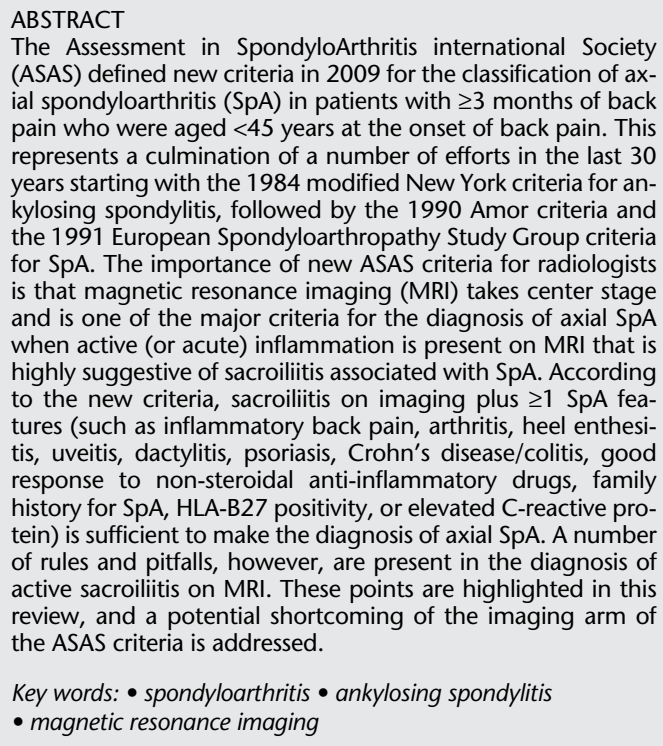

The Assessment in SpondyloArthritis international Society (ASAS) defined new criteria in 2009 for the classification of axial spondyloarthritis $(\mathrm{SpA})$ in patients with $\geq 3$ months of back pain who were aged $<45$ years at the onset of back pain. This represents a culmination of a number of efforts in the last 30 years starting with the 1984 modified New York criteria for ankylosing spondylitis, followed by the 1990 Amor criteria and the 1991 European Spondyloarthropathy Study Group criteria for SpA. The importance of new ASAS criteria for radiologists is that magnetic resonance imaging (MRI) takes center stage and is one of the major criteria for the diagnosis of axial SpA when active (or acute) inflammation is present on MRI that is highly suggestive of sacroiliitis associated with $\mathrm{SpA}$. According to the new criteria, sacroiliitis on imaging plus $\geq 1 \mathrm{SpA}$ features (such as inflammatory back pain, arthritis, heel enthesitis, uveitis, dactylitis, psoriasis, Crohn's disease/colitis, good response to non-steroidal anti-inflammatory drugs, family history for SpA, HLA-B27 positivity, or elevated C-reactive protein) is sufficient to make the diagnosis of axial SpA. A number of rules and pitfalls, however, are present in the diagnosis of active sacroiliitis on MRI. These points are highlighted in this review, and a potential shortcoming of the imaging arm of the ASAS criteria is addressed.

Key words: • spondyloarthritis • ankylosing spondylitis - magnetic resonance imaging

From the Department of Radiology (Ü.A. $\square$ ustunaydingoz@ yahoo.com, A.E.Y., S.A.Y., F.E., F.B.E.), Hacettepe University School of Medicine, Ankara, Turkey; the Department of Radiology (Z.M.Ö.), Inönü University School of Medicine, Malatya, Turkey.

Received 8 February 2012; accepted 8 February 2012.

Published online 6 April 2012

DOI 10.4261/1305-3825.DIR.5732-12.0
$\mathrm{S}$ pondyloarthritis (SpA) traditionally encompasses ankylosing spondylitis, psoriatic arthritis, arthritis associated with inflammatory bowel disease, arthritis associated with acute anterior uveitis, reactive arthritis, and undifferentiated SpA (1). In terms of clinical presentation, SpA may be predominantly axial (i.e., with involvement of the sacroiliac joints and/or the spinal column) or peripheral in location. The first decade of the new millennium saw significant progress in the management of axial spondyloarthritis in large part due to the use of tumor necrosis factor (TNF) antagonists in therapy. The objective verification of disease activity is even more important now, not only in terms of the initial diagnosis of SpA, but also for monitoring the response to treatment. Magnetic resonance imaging (MRI) can reveal pre-radiographic disease and may predict the subsequent development of ankylosing spondylitis, which is the characteristic SpA $(2,3)$. Early pre-radiographic patients with axial SpA may have active disease and pain that is as severe as that in those with established ankylosing spondylitis (4). Early pre-radiographic axial SpA responds well to antiTNF therapy (5).

The Assessment in SpondyloArthritis international Society (ASAS) defined new criteria in 2009 (Fig. 1) for the classification of axial SpA in patients with $\geq 3$ months of back pain who were aged $<45$ years at the onset of back pain (ASAS criteria for peripheral SpA have also been published recently) $(6,7)$. These criteria are the culmination of a number of efforts in the last 30 years starting with the 1984 modified New York criteria for ankylosing spondylitis, followed by the 1990 Amor criteria and the 1991 European Spondyloarthropathy Study Group criteria for SpA (8). The importance of 2009 ASAS criteria for radiologists is that MRI takes center stage in one of its two arms (Fig. 1). MRI is currently one of the major criteria for the diagnosis of axial SpA when active (or acute) inflammation that is highly suggestive of sacroiliitis associated with SpA is present on MRI. According to the new criteria (Fig. 1), sacroiliitis on imaging plus $\geq 1 \mathrm{SpA}$ features is sufficient to make the diagnosis of axial SpA.

\section{MRI features of active and chronic sacroiliitis}

Given the high diagnostic accuracy of MRI in determining joint and related bone marrow abnormalities, it is hardly surprising that MRI is now one of the cardinal tools for diagnosing active (acute) sacroiliitis associated with axial SpA (Fig. 1). We will first review the MRI criteria (according to ASAS) to make the diagnosis of active sacroiliitis (9) and mention the MRI criteria for chronic sacroiliitis (9). We will then proceed to how a sacroiliac MRI study should be tailored to address these criteria. Finally, we will discuss some differential diagnostic considerations, pitfalls, and a potential shortcoming of the imaging arm of the ASAS criteria. 


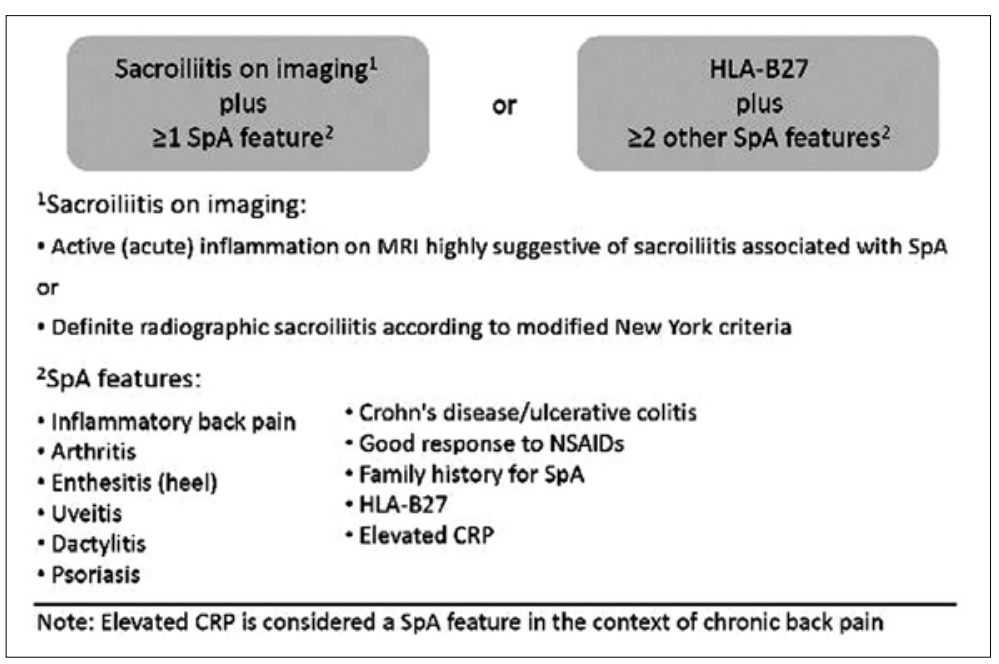

Figure 1. ASAS classification criteria for axial spondyloarthritis $(\mathrm{SpA})$ in patients with back pain $\geq 3$ months and age at onset $<45$ years (modified from reference 6).

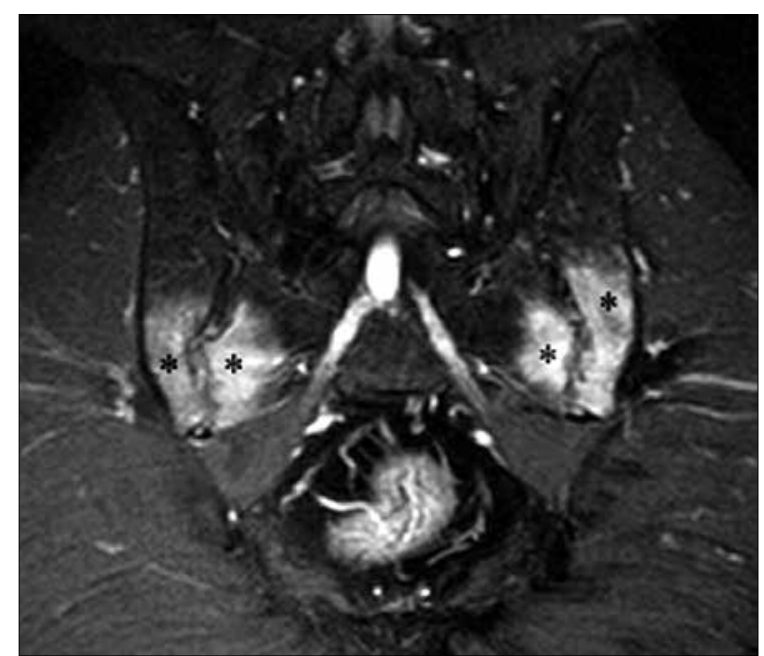

Figure 2. A coronal oblique STIR MR image shows bilateral periarticular bone marrow edema (asterisks) consistent with active sacroiliitis associated with axial SpA.
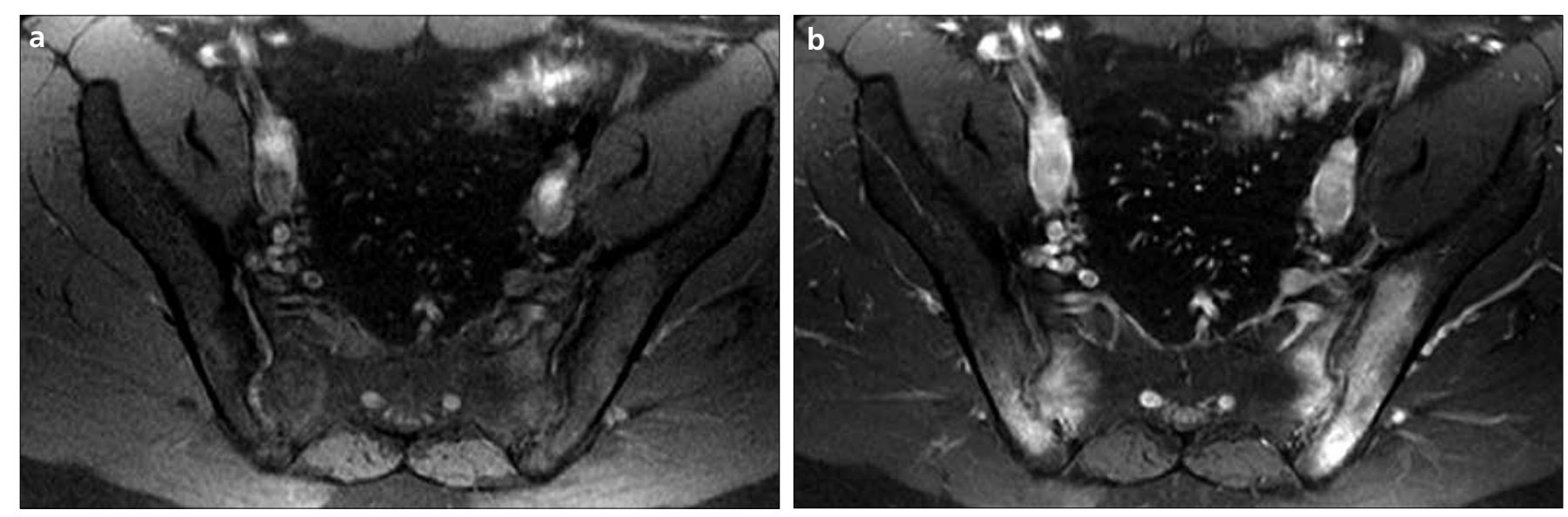

Figure 3. a, b. Fat-suppressed pre-contrast (a) and post-contrast (b) T1-weighted MR images show bilateral sacral and iliac-sided periarticular osteitis consistent with active sacroiliitis associated with axial SpA.

\section{MRI criteria for diagnosing active (acute)} sacroiliitis associated with axial SpA

There are four MRI findings of active (acute) sacroiliitis associated with axial SpA: osteitis/bone marrow edema, enthesitis, capsulitis, and synovitis. Of these four MRI findings, however, osteitis/bone marrow edema is the single indispensable criterion for the diagnosis of active sacroiliitis (9). In other words, the presence of enthesitis and/ or capsulitis and/or synovitis is not sufficient to make the MRI diagnosis of active sacroiliitis.

Osteitis/bone marrow edema is detected as a hyperintense signal on STIR (short tau inversion recovery) MRI sequence (Fig. 2) and typically as a hypointense signal on T1-weighted MRI sequences. When an area with this type of signal enhances with intravenous (i.v.) contrast, it is then called osteitis
(Fig. 3). The sacral interforaminal bone marrow signal constitutes the reference for a normal bone marrow signal. Although affected bone marrow areas are typically periarticular (subchondral) in location, there is no exact definition with respect to location. Osteitis/bone marrow edema is said to be "clearly present" for positive identification based on MRI (8); however, as with the location, no clear definition of the "intensity" of signal required is mentioned in the ASAS criteria.

The amount of signal required for a positive MRI for active (acute) sacroiliitis, however, is defined. If there is only one signal (suggesting osteitis/bone marrow edema) for each MRI slice, the lesion should be present on at least two consecutive slices. If there is more than one such signal on a single slice, one slice may be sufficient (9).
Enthesitis is seen as a hyperintense signal on STIR or contrast enhancement at sites where ligaments of the ligamentous portion of the sacroiliac joint attach to the sacrum or the iliac bone (Fig. 4). The abnormal signal may extend to the bone marrow and soft tissue.

Capsulitis is also depicted as a hyperintense signal on STIR or contrast enhancement at the anterior and posterior capsule (Fig. 4). Capsulitis may extend into the periosteum at the anterior capsule (Fig. 5) and may therefore essentially represent enthesitis as well.

Synovitis is best displayed as contrast enhancement on fat-suppressed T1weighted images in the synovial part of the sacroiliac joint space (Fig. 5). The signal intensity should be similar to blood vessels, but the configuration should not be similar. 

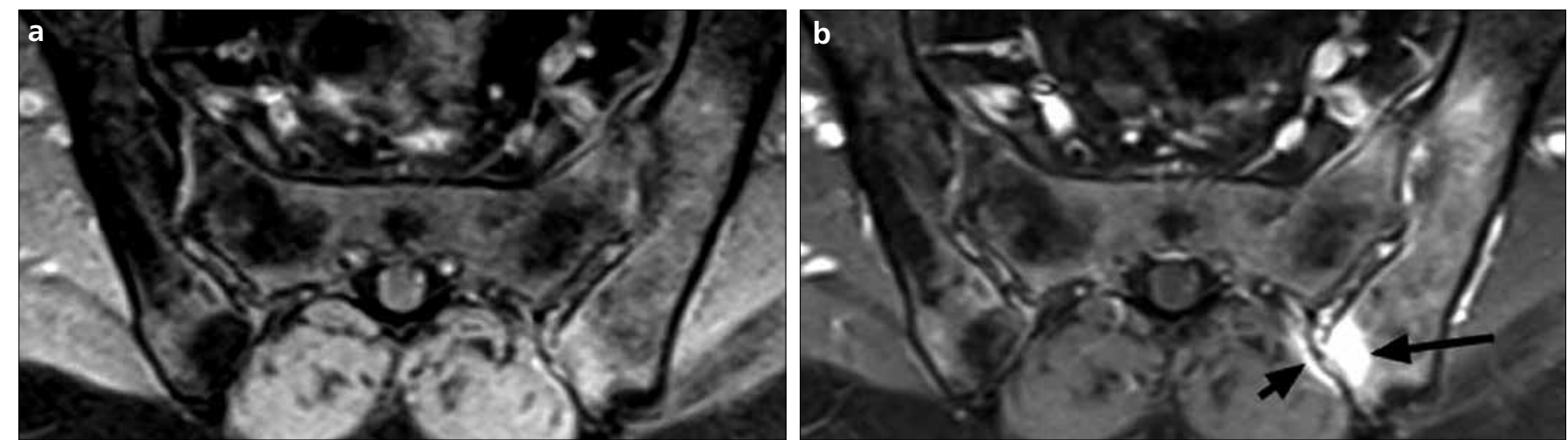

Figure 4. a, b. Transverse oblique fat-suppressed T1-weighted MR images before (a) and after (b) i.v. contrast show enthesitis (long arrow) and posterior capsulitis (short arrow).
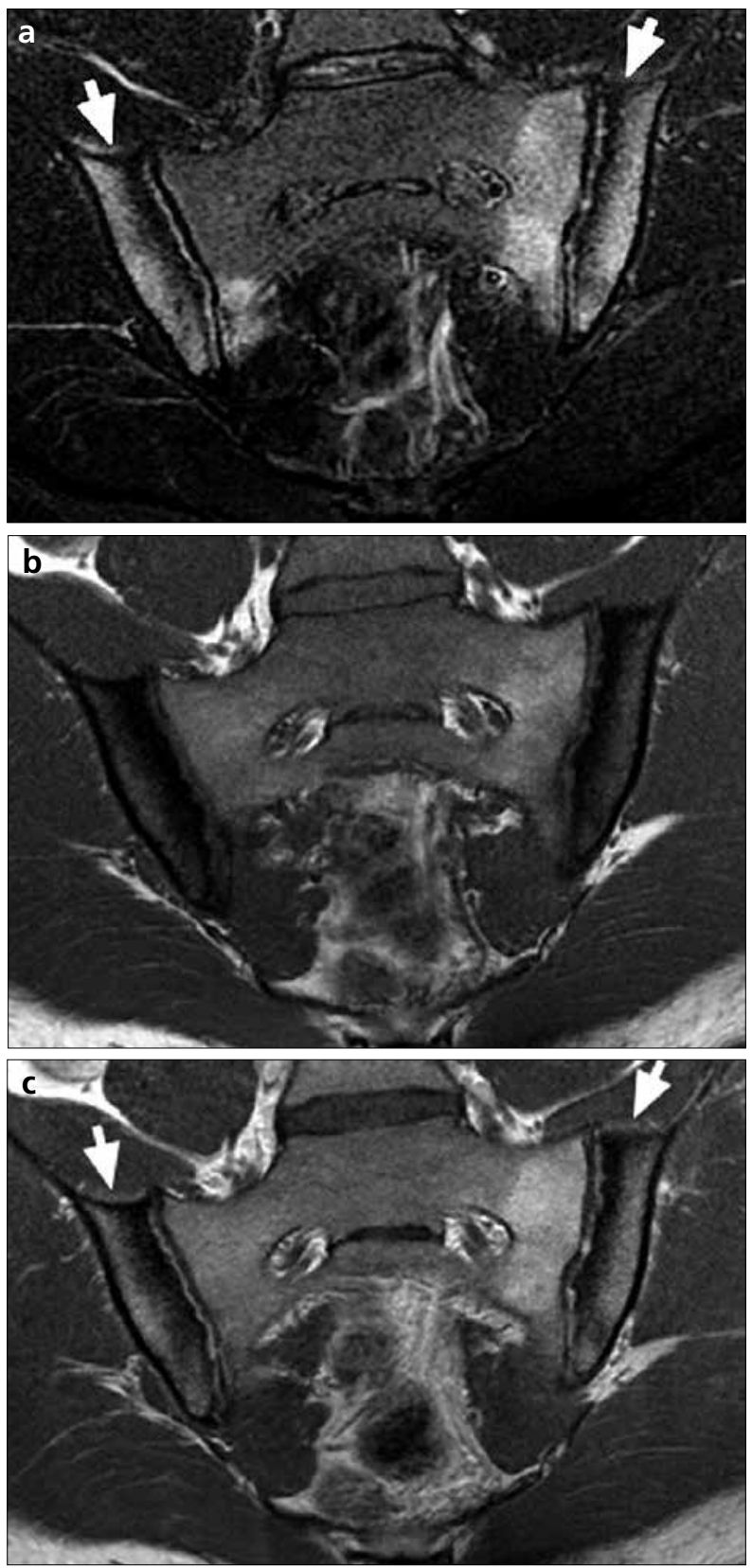

Figure 5. a-c. Coronal oblique STIR (a), T1-weighted pre-contrast (b) and T1-weighted postcontrast (c) MR images show sacroiliac synovitis (best visible on c), periarticular osteitis and anterior capsular enthesitis (arrows).

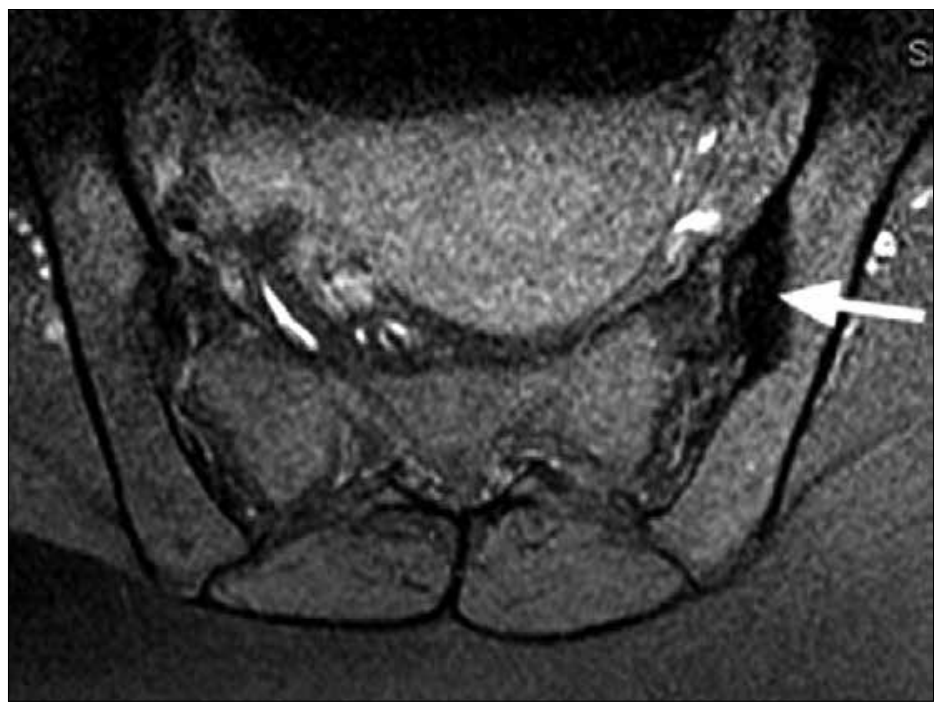

Figure 6. A transverse oblique fat-suppressed T1-weighted pre-contrast MR image shows subchondral sclerosis (arrow) of more than $5 \mathrm{~mm}$ in thickness.

MRI criteria for diagnosing chronic (structural damage) lesions of sacroiliitis associated with axial SpA

There are four types of lesions reflecting structural damage due to previous inflammation of the sacroiliac joints: subchondral sclerosis, subchondral/ periarticular erosions, periarticular fat deposition, and bony bridges/ankylosis. According to ASAS, the sole presence of structural lesions without concomitant osteitis/bone marrow edema is not sufficient for the definition of a positive MRI (9).

Subchondral sclerosis is defined as sclerotic areas that should extend at least $5 \mathrm{~mm}$ from the sacroiliac joint surface (Fig. 6); smaller areas of periarticular sclerosis may be physiological.

Subchondral/periarticular erosions are bony defects at the joint margin (Fig. 7). They may unite with each other to result in pseudowidening of the sacroiliac joints (Fig. 7). 

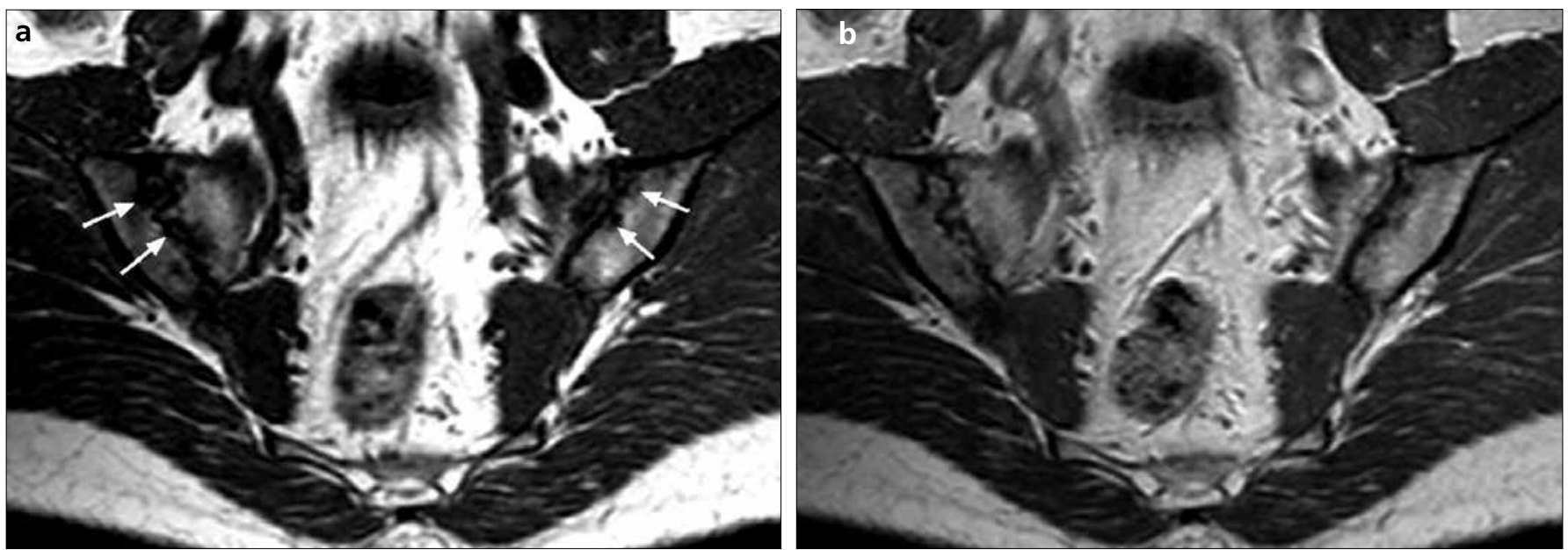

Figure 7. a, b. Coronal oblique T1-weighted MR images before (a) and after (b) i.v. contrast show erosions (arrows), the confluence of which has resulted in the pseudo-widened appearance of the right sacroiliac joint. Note that the erosions are more on the iliac, rather than the sacral, sides of the joints because the articular cartilage overlying the iliac side is half as thin as that on the sacral side.
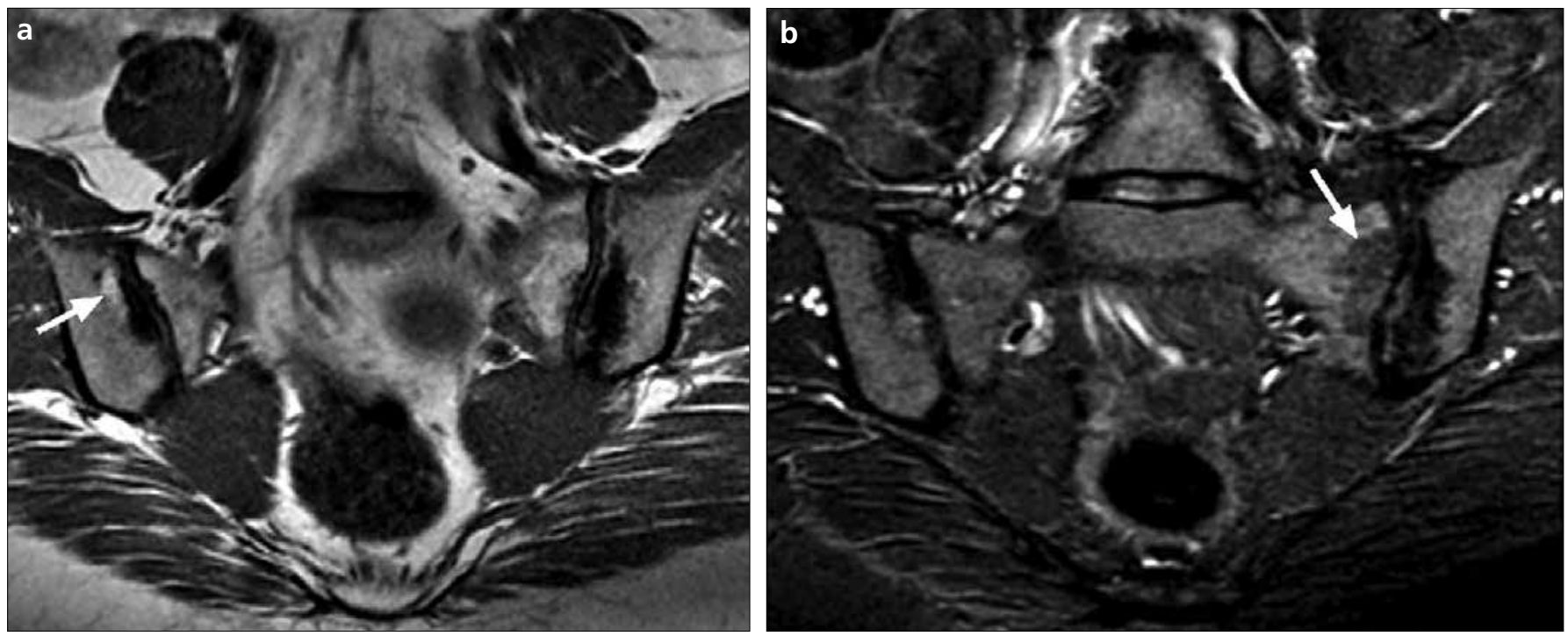

Figure 8. a, b. Coronal oblique T1-weighted pre-contrast (a) and STIR (b) MR images show periarticular fat deposition (arrows) on both sides. Note the iliac-sided subchondral sclerosis adjacent to the left sacroiliac joint.

Periarticular fat deposition most likely indicates areas of previous inflammation where the esterification of fatty acids has occurred (Fig. 8). No further definition of "periarticular," however, has been made (9), and this lack of further definition predisposes some, if not many, lesions to ambiguity.

Bony bridges/ankylosis typically results from the fusion of bone buds that have formed during the course of inflammation and face each other. This may be focal or extensive (Fig. 9).

All structural damage lesions can be displayed by MRI. However, with the exception of periarticular fat deposition, which is best displayed by MRI, their evaluation is typically carried out on direct radiographs by clinicians. It is important for radiologists to point out to clinicians that MRI is very useful to detect chronic (structural damage) as well as acute sacroiliac lesions in axial SpA (Fig. 10). Although direct radiography is readily available and inexpensive, its days of extensive use in the evaluation of sacroiliitis may be numbered. Either negative or positive direct radiographs do not exclude active sacroiliitis. Disease activity is best displayed by MRI, and it is an important parameter in the tailoring and follow-up of treatment. From a practical standpoint, the level of expertise required for the correct evaluation of direct radiography for sacroiliitis is higher and less readily available than that required for the proper evaluation of MRI of the sacroiliac joints. In light of these issues, we propose that the abandonment of direct radiography in the routine work-up of sacroiliitis should be seriously considered.

\section{Heel enthesitis}

Enthesitis elsewhere in the body such as in the heel can be easily verified by MRI (Fig. 11). STIR and T1weighted long axis and fat-suppressed T1-weighted short axis MRI sequences (before and after i.v. contrast) would readily display enthesitis. Because heel enthesitis is among the SpA features, it is important for radiologists to increase awareness on the side of the clinicians (e.g., rheumatologists) of this capability of MRI. 

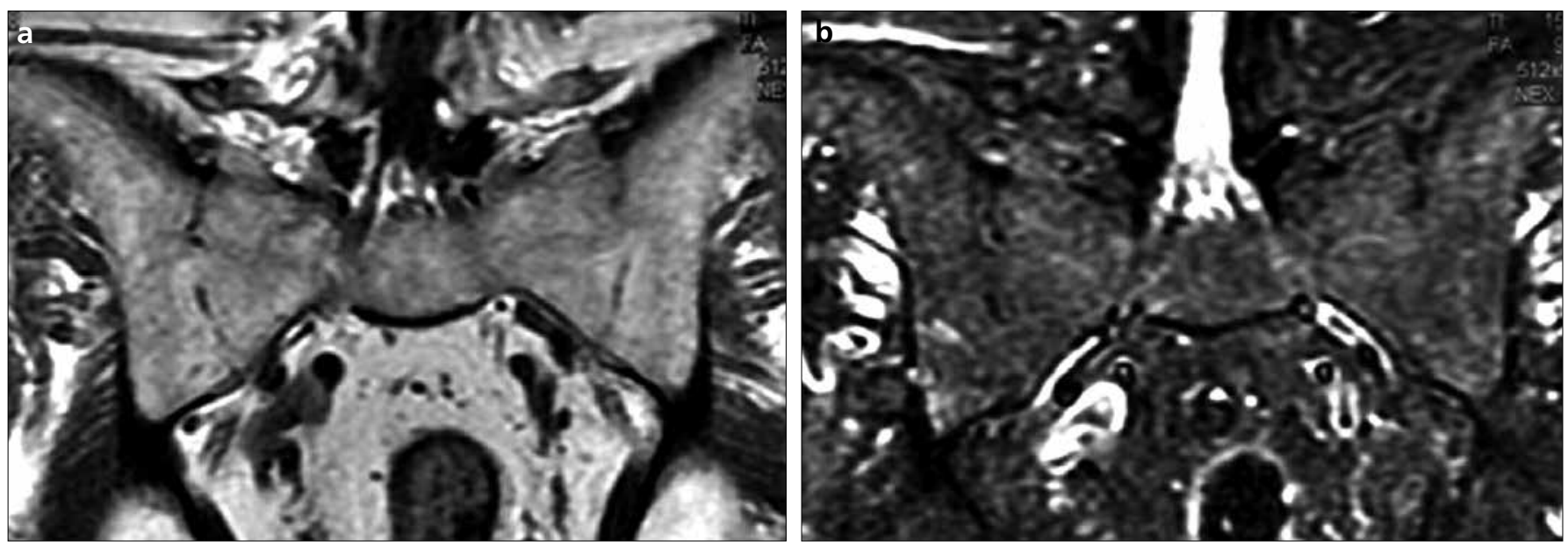

Figure 9. a, b. Coronal oblique T1-weighted pre-contrast (a) and STIR (b) MR images show bony bridging (in the form of near complete ankylosis) in both sacroiliac joints. The patient has ankylosing spondylitis (without active sacroiliitis).
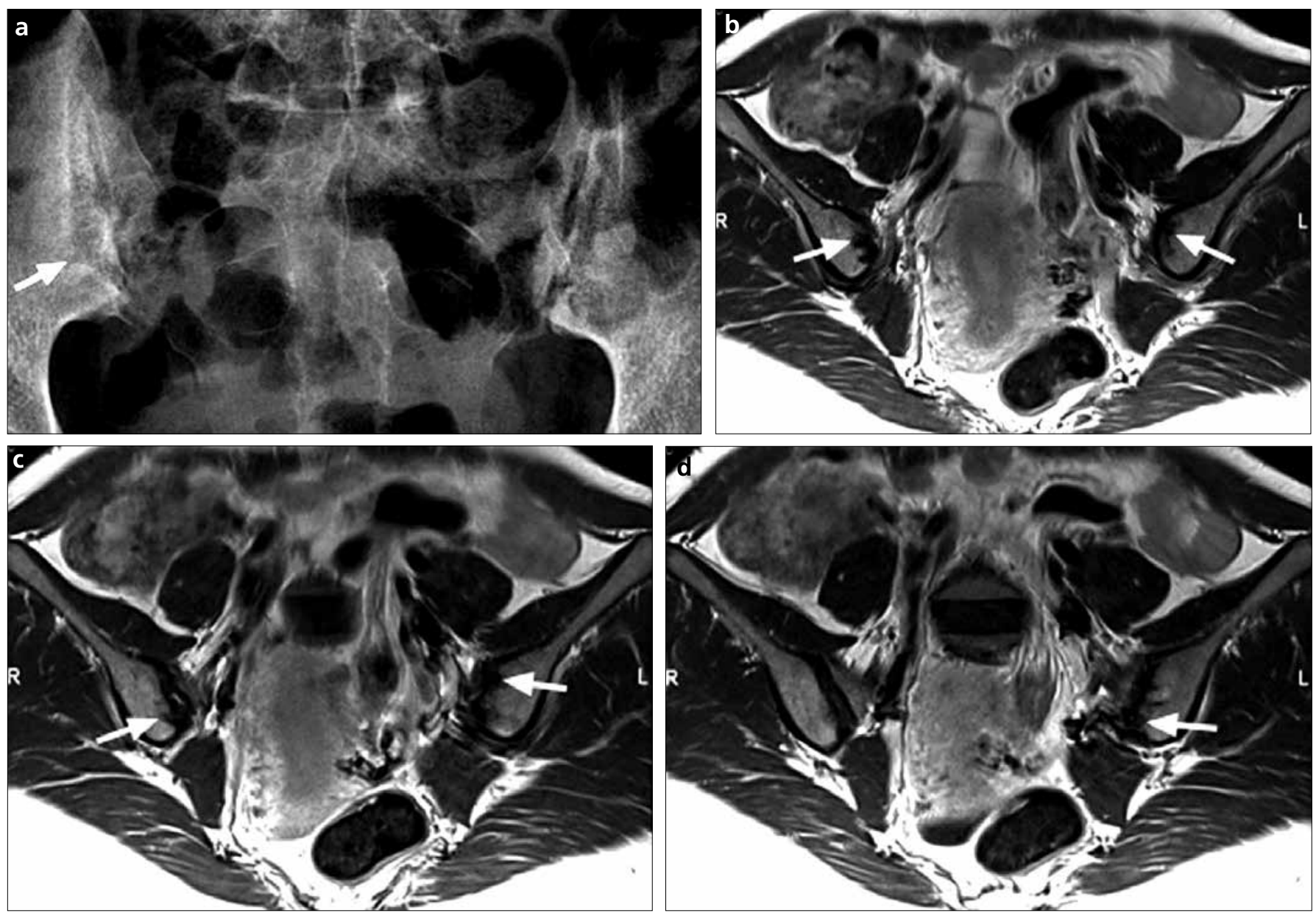

Figure 10. a-d. Direct radiography (Ferguson view, a) shows erosions (arrow) at the iliac side of the right sacroiliac joint. Coronal oblique post-contrast T1-weighted MR images (b-d) depict erosions (arrows) at the iliac sides of both sacroiliac joints. Direct radiography was obtained 20 days before MRI.

Tailoring an MRI examination of the sacroiliac joints to enable evaluation based on the ASAS criteria

Imaging sacroiliac joints on two planes that are perpendicular to each other (transverse oblique and coronal oblique; Fig. 12) would ensure the adequate display of as much cross-sectional interface through these joints as possible in a reasonable amount of time. Administering i.v. gadoliniumbased contrast material will help with the detection of subtle osteitis/bone marrow edema, enthesitis, capsulitis, and synovitis.

A typical MRI of the sacroiliac joints would therefore have the following sequences: 


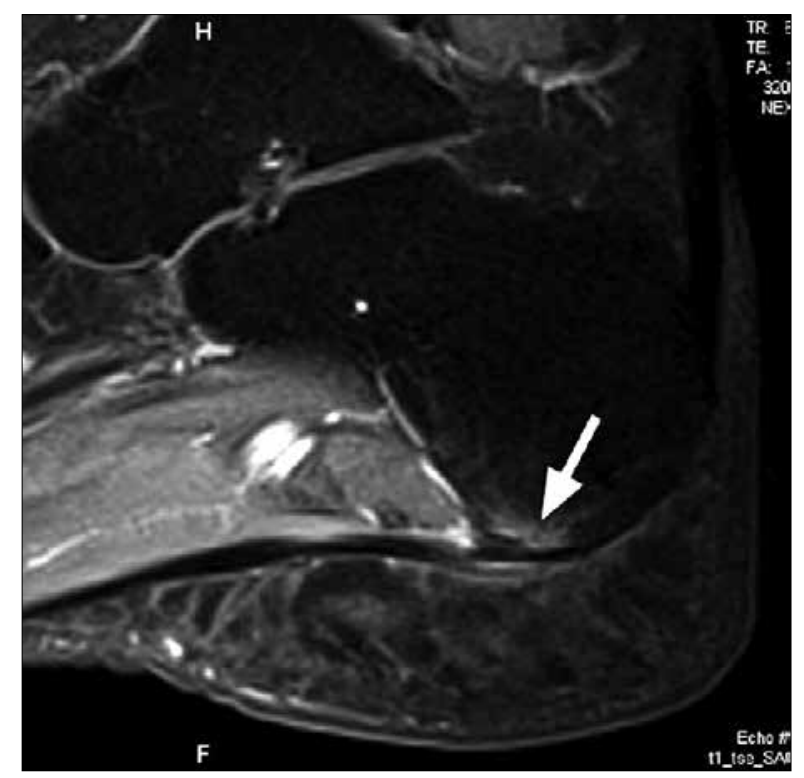

Figure 11. Heel enthesitis (arrow) displayed as contrast enhancement of the plantar fascia enthesis on a postcontrast fat-suppressed T1weighted MR image.

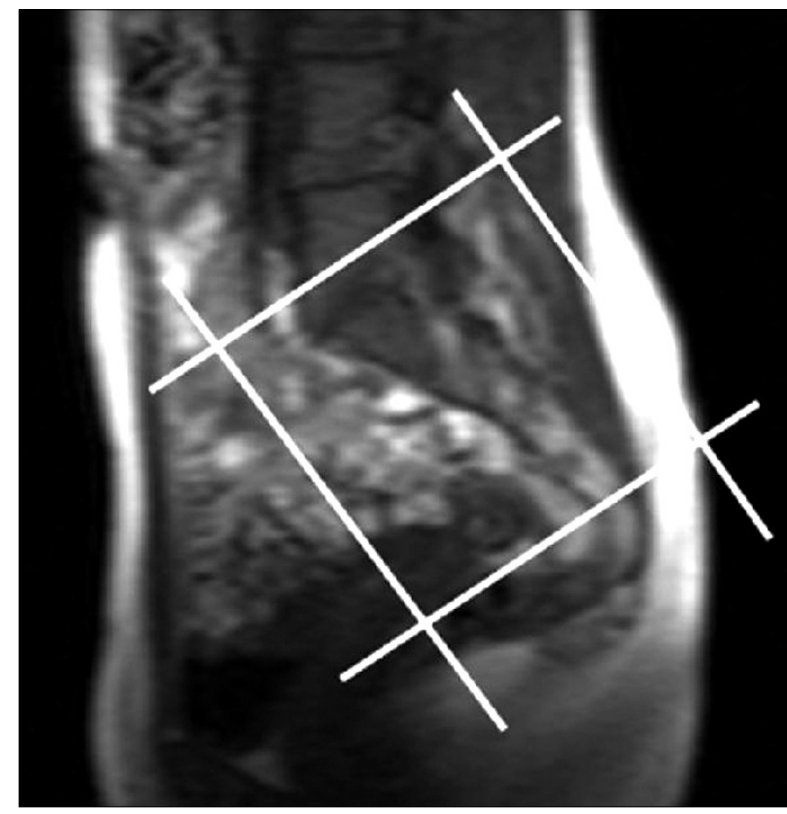

Figure 12. Approximate locations of extreme slices of the coronal oblique and transverse oblique sacroiliac MRI sequences on a scout image.

1. STIR and T1-weighted coronal oblique (perpendicular to the S1 superior end-plate and covering the entire sacroiliac joints)

2. Fat suppressed T1-weighted (before and after i.v. contrast) transverse oblique (parallel to the S1 superior end-plate and covering the entire sacroiliac joints)

Given the possibility of aborting the MRI exam for any reason (typically due to patient intolerance because of claustrophobia), it is prudent to obtain the STIR and T1-weighted coronal oblique sequences first, as one or both of these two would provide important information on the presence or absence of osteitis/bone marrow edema, which is essential in terms of the clinical concern (i.e., whether there is active sacroiliitis). In patients where i.v. contrast is contraindicated, the fat-suppressed T1weighted sequence should be replaced with the STIR transverse oblique sequence. It is important to remember that STIR provides much more uniform fat suppression than-and should therefore be favored overfat-suppressed T2-weighted sequence; although, admittedly, the former has a non-specific suppression when compared to the latter (in other words, with STIR, signal from tissue or fluidsuch as mucoid tissue, hemorrhage, proteinaceous fluid, gadolinium or melanin in a given concentrationwith a T1 similar to that of fat will also be suppressed) (10).

With a $512 \times 512$ image matrix and two signal acquisitions, it is typically possible to obtain images that would provide adequate information on the presence of erosions. Such information, in our practice, is at least consistently equal to or typically better than that provided by direct radiography (i.e., Ferguson view) (Fig. 10). Other chronic (structural damage) lesions (namely, subchondral sclerosis, periarticular marrow fat deposition, and ankylosis) are always better depicted by MRI than direct radiography because of the former's cross-sectional capability and superb bone marrow imaging properties. Overall, in our practice, MRI is sufficient to show the entire active (acute) and structural damage (chronic) lesions associated with axial spondyloarthritis.

\section{Differential diagnostic considerations for active sacroiliitis on MRI}

Inflammation of the sacroiliac joints due to infection (septic sacroiliitis) crosses anatomical borders, but SpA does not. Septic sacroiliitis spreads diffusely to adjacent soft tissues (Fig. 13).

Insufficiency fractures typical of the sacrum (Fig. 14), and bone tumors such as plasmacytoma and osteosarcoma can cause a bone marrow edema/ osteitis-like appearance on MRI.

Osteoarthritis of sacroiliac joints may occasionally be associated with small areas of bone marrow edema along the sacroiliac joint. With osteoarthritis, it is not uncommon to see anterior osteophytes surrounding the sacroiliac joints. Such osteophytes may be seen as areas of sclerosis on a direct radiograph; however, their true nature will be readily displayed on the crosssectional images of MRI (Fig. 15).

Osteitis condensans ilii (Fig. 16) has a typical location and configuration on MRI as on direct radiography/computed tomography. It is typically seen in middle-aged women in whom it has been attributed to abnormal stresses associated with pregnancy and delivery.

\section{Pitfalls of active sacroiliitis on MRI}

Blood vessels crossing through the sacroiliac joints may be mistaken for active inflammation on STIR images (Fig. 17). Consecutive images should be carefully examined to rule out this possibility. 

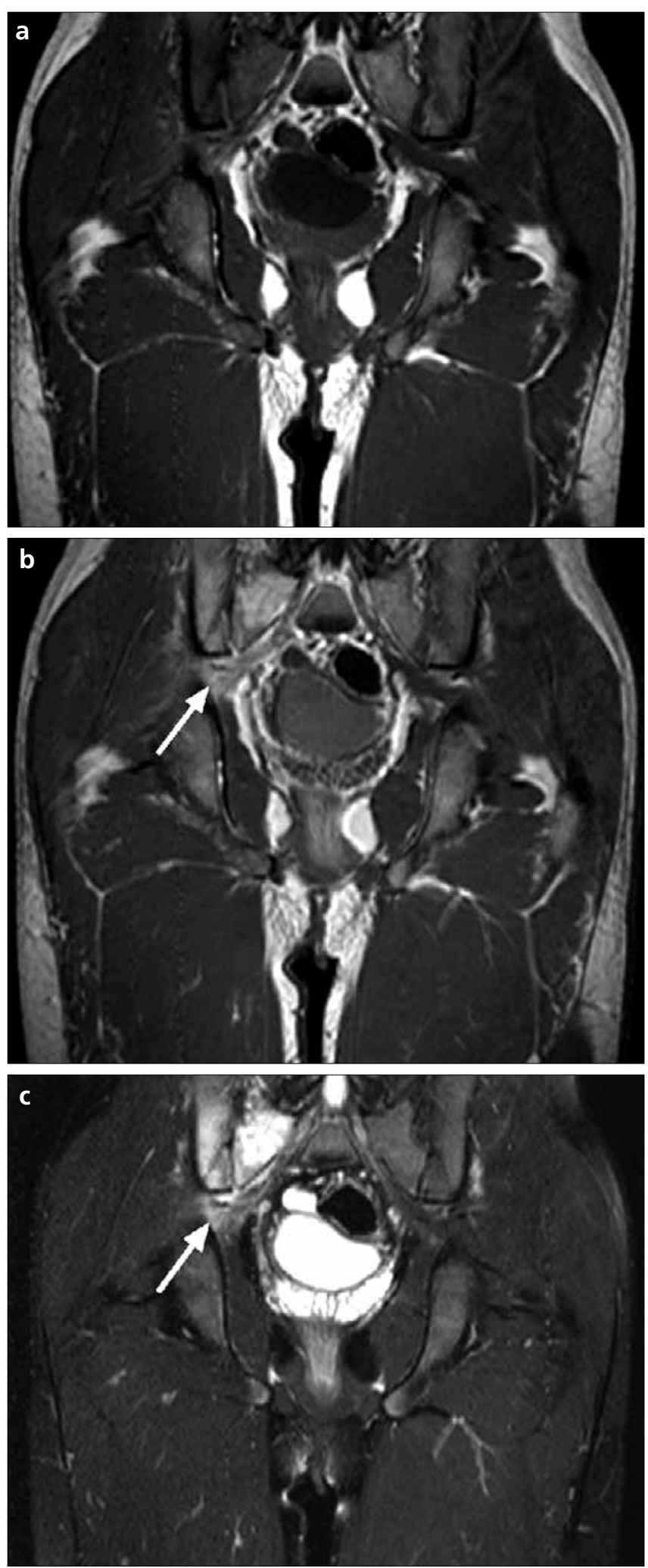

Figure 13. a-c. Coronal T1-weighted pre-contrast (a), postcontrast (b), and STIR (c) MR images show periarticular osteitis and adjacent soft tissue inflammation (arrows) in a patient with right septic sacroiliitis. The exam was planned to address rightsided hip pain.
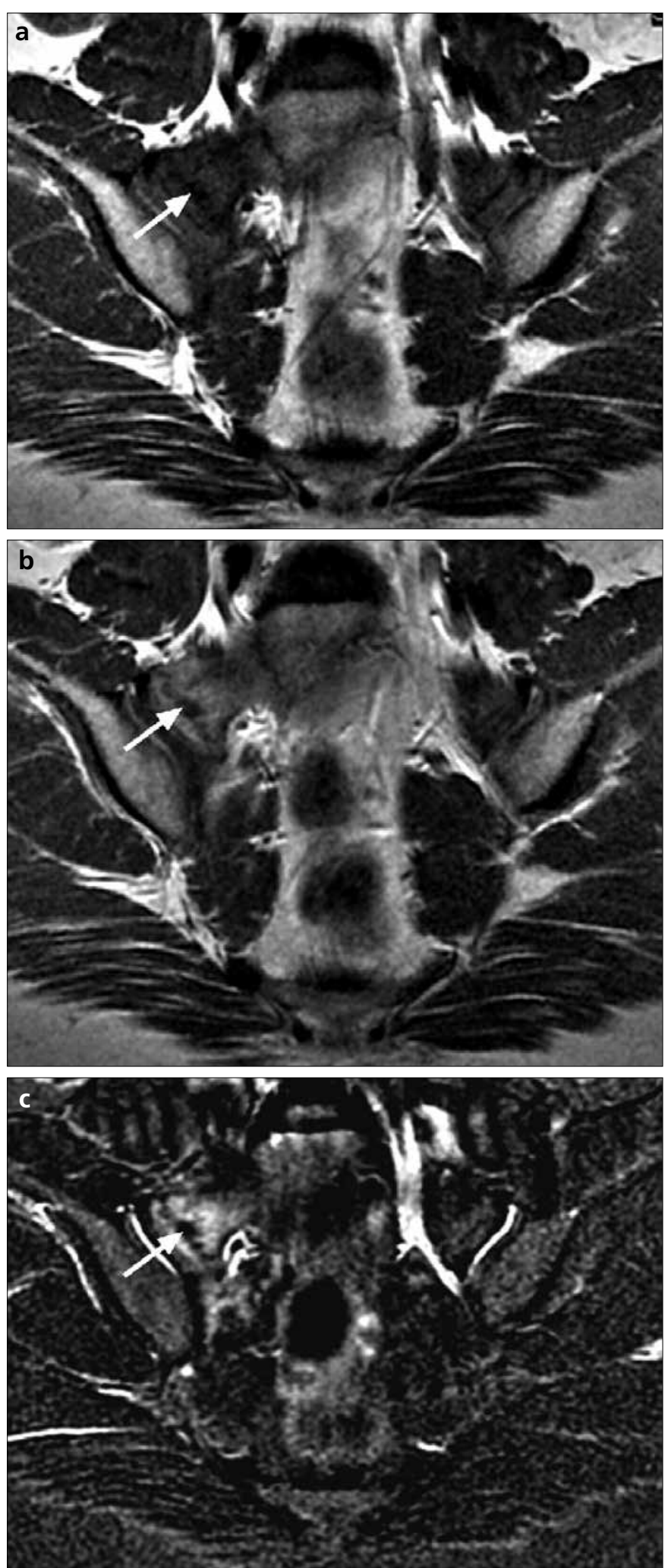

Figure 14. a-c. Coronal oblique pre-contrast (a) and post-contrast (b) T1-weighted and STIR (c) MR images show an insufficiency fracture (arrows) of the right sacral ala with surrounding bone marrow edema in a patient under treatment for Hodgkin lymphoma. 

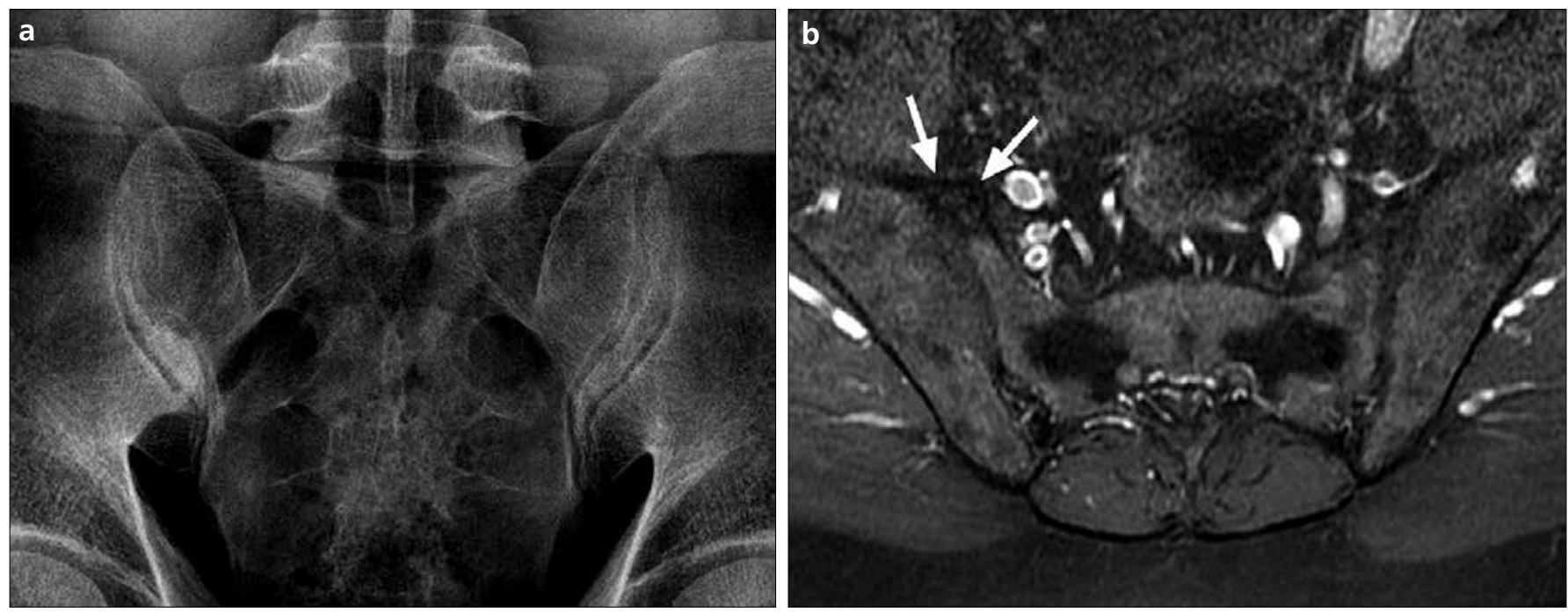

Figure 15. a, b. Direct radiography (Ferguson view) (a) show areas of sclerosis in the region of the right sacroiliac joint, which turned out to be axuberant anterior osteophytes (arrows) on a transverse oblique fat-suppressed post-contrast T1-weighted MR image (b).
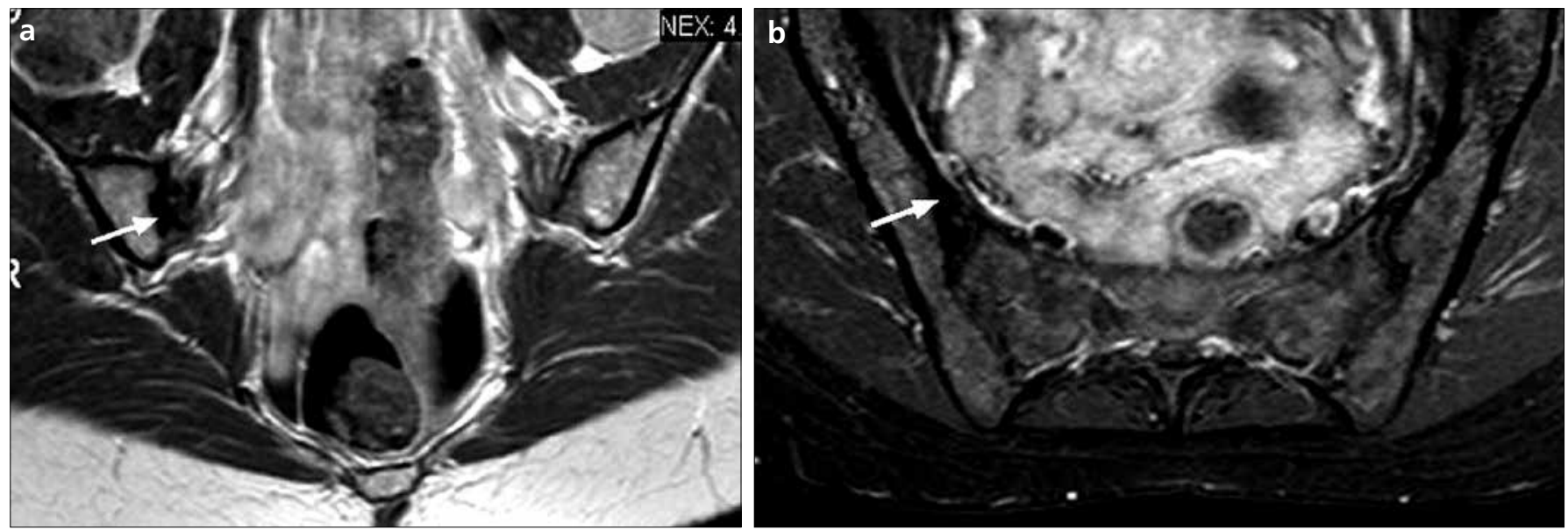

Figure 16. a, b. Coronal oblique T1-weighted (a) and transverse oblique fat-suppressed T1-weighted (b) post-contrast MR images show subchondral sclerosis (arrows) at the iliac side of the right sacroiliac joint in a triangular configuration with a base at the bone margin inferiorly and anteriorly (osteitis condensans ilii). The patient is a 42-year-old multiparous woman.

Inadequate fat suppression (typically seen with T2-weighted spectral fat suppression; not with STIR) may cause normal anatomical structures to appear as hyperintense, especially at the posterior part of the sacrum and sometimes of the iliac bone. Additionally, the so-called "coil effect" or "coil burn-out" may result in brighter signal closer to the coil-body interface (Fig. 18). Similar effects on the adjacent soft tissue help to distinguish these conditions from real alterations.

Pulsation artifacts from the pelvic/ iliac vessels may be juxtaposed to the otherwise normal bone marrow and may be mistaken for osteitis/bone marrow edema (Fig. 19). Alternatively, they may mask underlying osteitis/ bone marrow edema.
Finally, it should be borne in mind that children have incompletely ossified bones and the chondral interface between the ossified portions of the sacrum or iliac bone and the sacroiliac joint margin may resemble bone marrow edema (Fig. 20).

\section{A potential shortcoming of the imaging arm of the ASAS criteria for diagnosing axial SpA}

Of the ten people that participated in the study to define "active sacroiliitis on MRI for the classification of axial spondyloarthritis" that formed one of the bases of the new ASAS criteria, only two were radiologists (the remainder were rheumatologists) (9). In daily practice, many rheumatologists apparently feel comfortable evaluating MRI of the sacroiliac joints themselves. It is not surprising that a properly conducted MRI provides quite objective cross-sectional information on axial SpA "for all to see". However, in regard to the definition and establishment of MRI criteria, the vast experience of musculoskeletal radiologists must be tapped accordingly.

The wording of ASAS classification criteria for axial SpA has it that sacroiliitis on imaging is either in the form of "active (acute) inflammation on MRI highly suggestive of sacroiliitis associated with SpA" or "definite radiographic sacroiliitis according to modified New York criteria" (Fig. 1). The ASAS criteria may potentially be used by family physicians, general practitioners, internal medicine specialists, as well as rheumatologists. It is obvious that 

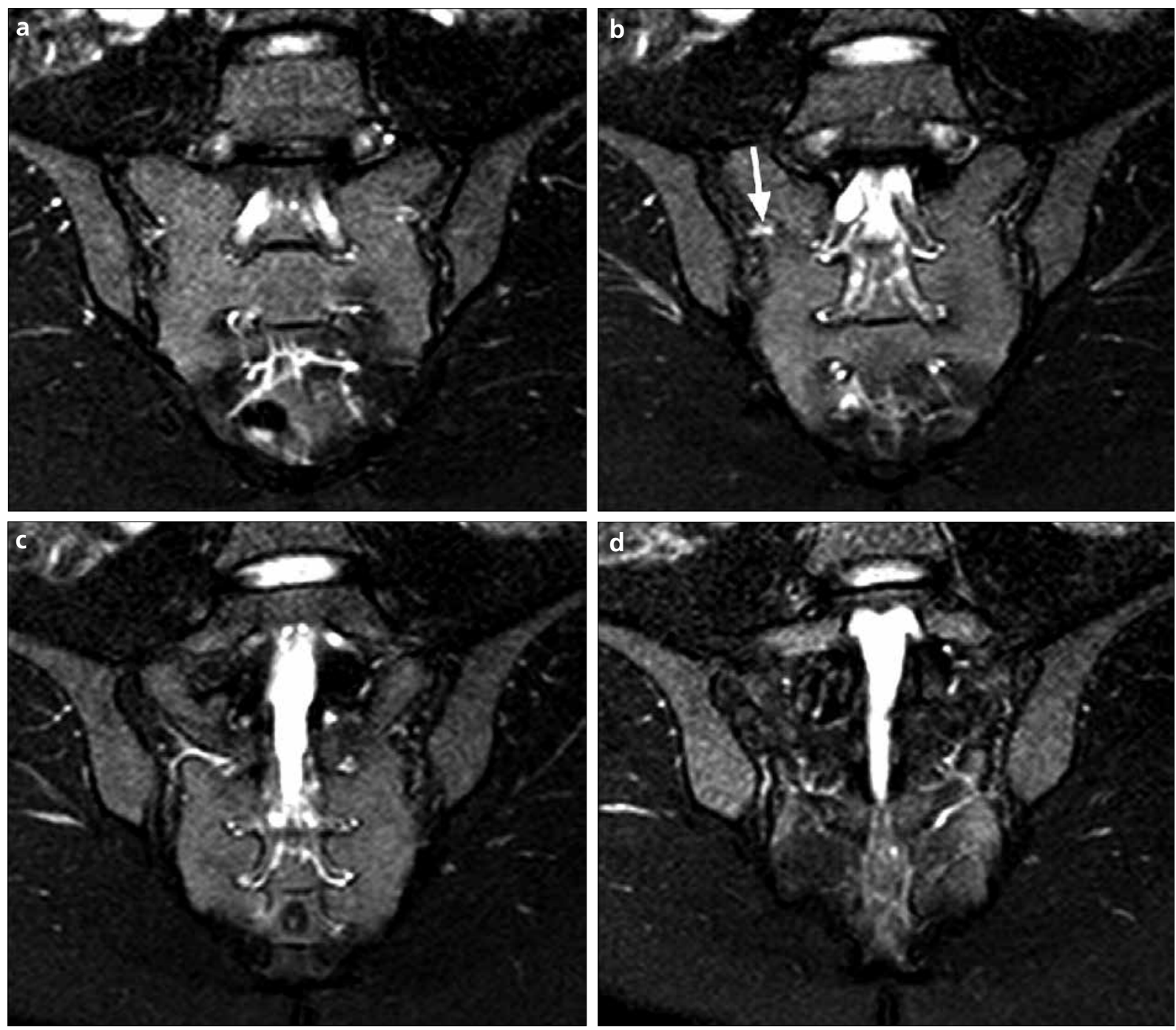

Figure 17. a-d. What appears to be focal bone marrow edema/osteitis on the subchondral sacral side of the right sacroiliac joint (arrow, b) turns out to be a crossing vessel on serial coronal oblique STIR MR images.
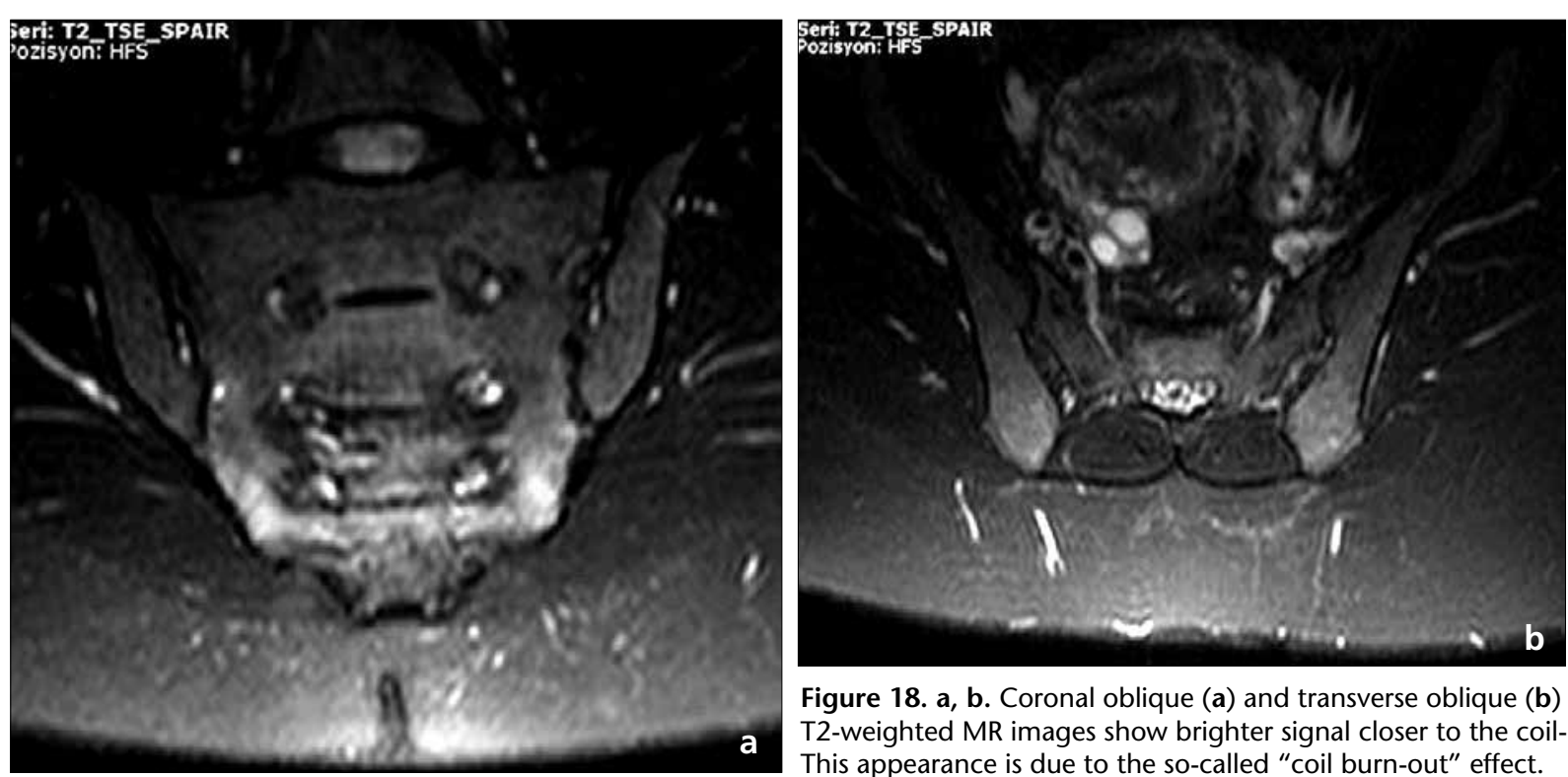

Figure 18. a, b. Coronal oblique (a) and transverse oblique (b) fat-suppressed T2-weighted MR images show brighter signal closer to the coil-body interface. This appearance is due to the so-called "coil burn-out" effect. 

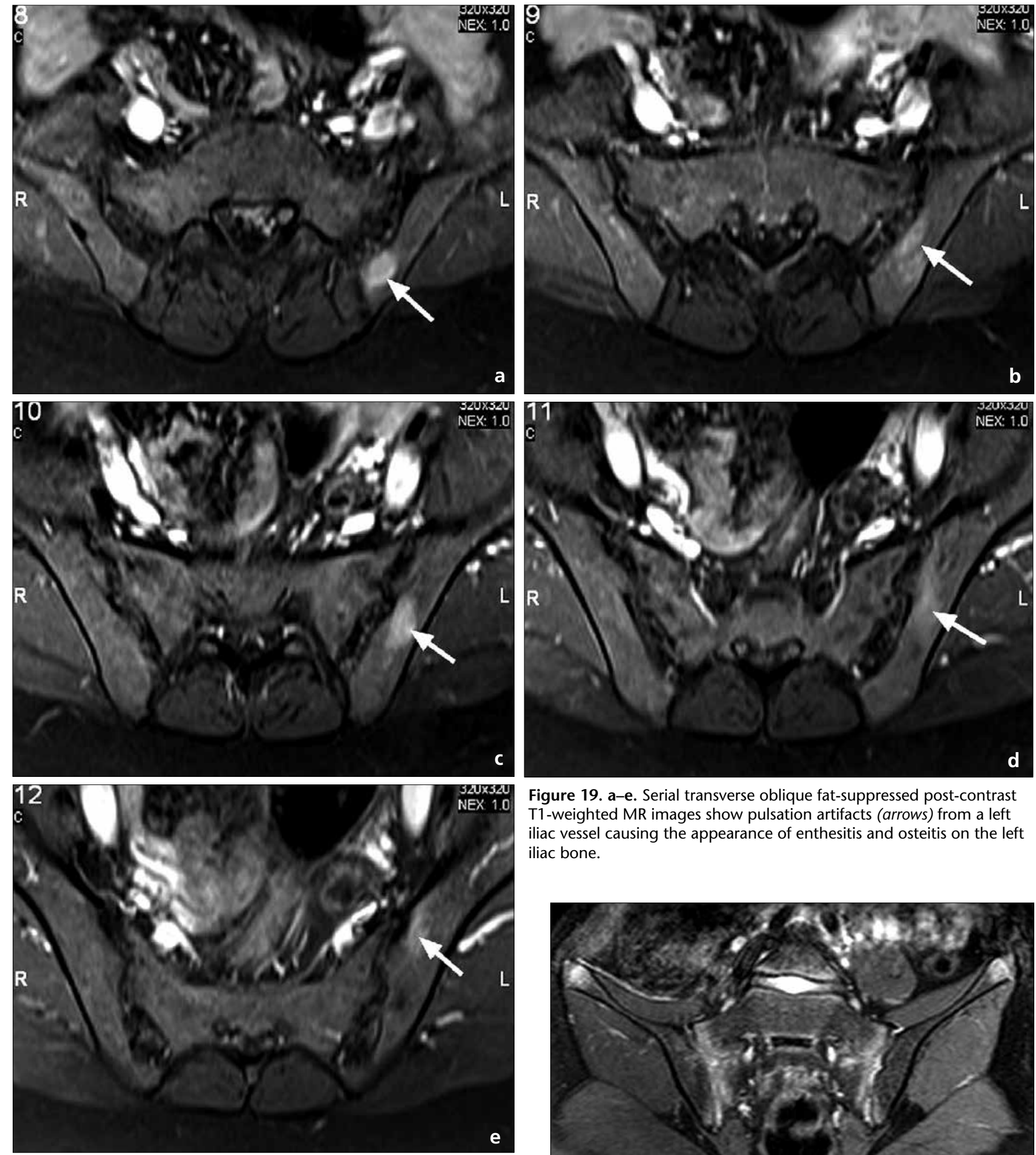

Figure 19. a-e. Serial transverse oblique fat-suppressed post-contrast T1-weighted MR images show pulsation artifacts (arrows) from a left iliac vessel causing the appearance of enthesitis and osteitis on the left iliac bone.

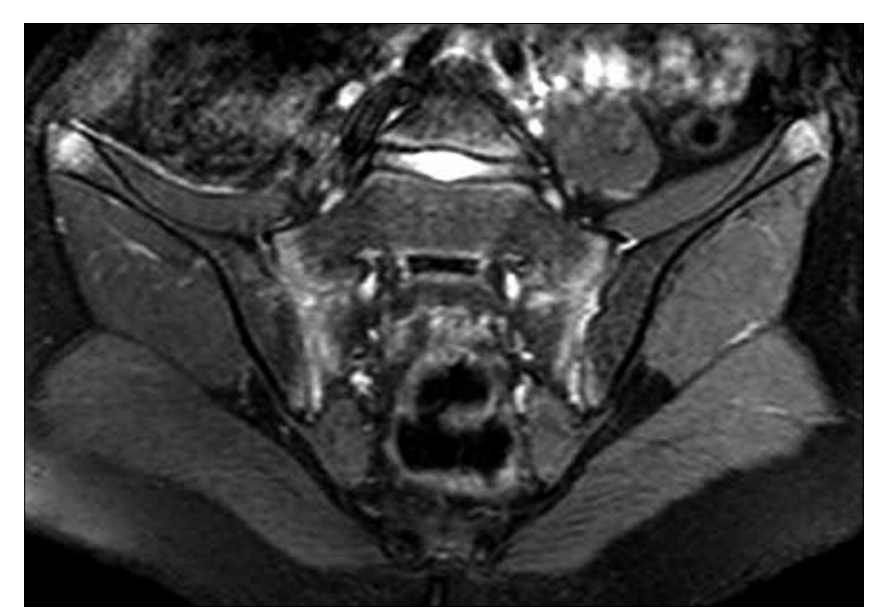

Figure 20. A coronal oblique fat-suppressed T2-weighted MR image depicts symmetrical unossified cartilage of the iliac bones and sacrum in a 14-year-old boy. Note the fluid-like interface at the sacroiliac joint margins. Also note poor fat suppression at the right buttock subcutaneous tissue (this is not a STIR image). 
most rheumatologists would not be content with a negative direct radiography examination, which is currently the initial imaging step for diagnosing axial SpA; many will likely go on to order MRI of the sacroiliac joints in the face of compelling clinical suspicion of SpA. The same does not need to be true, however, for non-rheumatologists, who may inadvertently perceive the wording of the ASAS criteria to indicate that "radiography negative for sacroiliitis" means "imaging negative for sacroiliitis". The wording of the criteria is rather vague in this regard and may easily be misinterpreted by an inexperienced non-rheumatologist to mean that "when you have a positive MRI for active (acute) sacroiliitis that is a cardinal feature; however, when you do not have an MRI at all, and if you have negative direct radiography, imaging is negative". In other words, in the absence of MRI of the sacroiliac joints, the presence of a direct radiography exam negative for sacroiliitis should not hold back or defer the clinician from ordering an MRI. Whereas a positive MRI means the presence of "active inflammation", and structural damage lesions do not count for a positive MRI according to the ASAS, the very inclusion of "definite radiographic sacroiliitis according to modified New York criteria" as the other indicator of "sacroiliitis on imaging" is rather contradictory, especially in light of the fact that all structural damage lesions-of definite radiographic sacroiliitis-can be unambiguously shown by a properly performed MRI.

A recent study performed to validate the ASAS criteria and define positive MRI of the sacroiliac joint in an inception cohort of axial spondyloarthritis followed up for eight years found that both arms of the ASAS criteria have good diagnostic utility in early SpA, although they are of limited value for the prediction of radiographic progression (11). The authors stated that a full reappraisal of the definition of what constitutes a positive MRI for sacroiliitis in SpA, which would have prognostic as well as diagnostic relevance, is required (11). The authors proposed that the calibration of the severity of the bone marrow edema MRI signal and the inclusion of structural changes as evidenced on MRI may be considered (11). Such a possibility would entail considering the chronic, as well as active (acute), sacroiliitis features of MRI in the assessment of patients with established or possible SpA. Perhaps, a grading scale for global sacroiliitis features of MRI may be developed in this regard. We believe that MRI is already able to do just that.

\section{Conclusion}

STIR and T1-weighted coronal oblique (perpendicular to the S1 superior end-plate and covering the entire sacroiliac joints) and fat-suppressed $\mathrm{T} 1$ weighted (before and after i.v. contrast) transverse oblique (parallel to the S1 superior end-plate and covering the entire sacroiliac joint) MRI sequences should be used in sacroiliac joint imaging. In patients where i.v. contrast is contraindicated, fat-suppressed T1-weighted MRI sequence should be replaced with STIR transverse oblique sequence.

Osteitis/bone marrow edema is the single indispensable MRI finding of active sacroiliitis. It should be noted, however, that MRI is capable of showing all established imaging findings of active and chronic sacroiliitis. The fact that MRI has now taken center stage in the diagnosis of axial spondyloarthropathy is a tribute to this modality's extensive ability to reveal joint and bone marrow abnormalities. Radiologists should play an active role in the workup of patients suspected to have active sacroiliitis. The close collaboration of radiologists with rheumatology and physical therapy and rehabilitation experts would result in mutual benefits for radiologists and clinicians, and it would result in improved patient care.

The wording of the ASAS criteria should be revised such that resortingby inexperienced clinicians-to direct radiography alone in terms of imaging must be avoided. When direct radiography is negative, MRI in patients who are clinically suspected to have acute or chronic sacroiliitis associated with axial SpA is still necessary. Given the lack of widespread expertise in the direct radiographic evaluation of sacroiliac joints and the ability of MRI to readily depict acute and chronic sacroiliitis, we propose that the possibility of abandoning direct radiographs altogether in the diagnostic work-up and follow-up of sacroiliitis associated with SpA should be seriously contemplated.

The increased participation of radiologists in committees responsible for developing imaging criteria for sacroiliitis is necessary. This would help fine-tune the establishment and description of such criteria.

\section{Conflict of interest disclosure}

The authors declared no conflicts of interest.

\section{References}

1. van den Berg R, van der Heijde DM. How should we diagnose spondyloarthritis according to the ASAS classification criteria: a guide for practicing physicians. Pol Arch Med Wewn 2010; 120:452-457.

2. Bennett AN, Marzo-Ortega $\mathrm{H}$, Emery $\mathrm{P}$, McGonagle D, on behalf of the Leeds Apondyloarthropathy Group. Diagnosing axial spondyloarthropathy. The new Assessment in SpondyloArthritis international Society criteria: MRI entering centre stage. Ann Rheum Dis 2009; 68:765-767.

3. Bennett AN, McGonagle D, O'Connor P, et al. Severity of baseline magnetic resonance imaging-evident sacroiliitis and HLA-B27 status in early inflammatory back pain predict radiographically evident ankylosing spondylitis at eight years. Arthritis Rheum 2008; 58:3413-3418.

4. Rudwaleit M, Haibel H, Baraliakos X, et al. The early disease stage in axial spondylarthritis: results from the German Spondyloarthritis Inception Cohort. Arthritis Rheum 2009; 60:717-727.

5. Barkham N, Keen $\mathrm{H}$, Coates L, et al. A randomized controlled trial of infliximab shows clinical and MRI efficacy in patients with HLA-B27 positive very early ankylosing spondylitis. Arthritis Rheum 2007; 56(Suppl):L11.

6. Rudwaleit M, van der Heijde D, Landewé $\mathrm{R}$, et al. The development of Assessment of SpondyloArthritis international Society classification criteria for axial spondyloarthritis (part II): validation and final selection. Ann Rheum Dis 2009; 68:777-783.

7. Machado P, Castrejon I, Katchamart W, et al. Multinational evidence-based recommendations on how to investigate and follow-up undifferentiated peripheral inflammatory arthritis: integrating systematic literature research and expert opinion of a broad international panel of rheumatologists in the 3E Initiative. Ann Rheum Dis 2011; 70:15-24.

8. Sieper J, Rudwaleit M, Baraliakos X, et al. The Assessment of SpondyloArthritis international Society (ASAS) handbook: a guide to assess spondyloarthritis. Ann Rheum Dis 2009; 68(Suppl II):ii1-ii44.

9. Rudwaleit M, Jurik AG, Hermann K-GA, et al. Defining active sacroiliitis on magnetic resonance imaging (MRI) for classification of axial spondyloarthritis: a consensual approach by the ASAS/OMERACT MRI group. Ann Rheum Dis 2009; 68:1520-1527.

10. Delfaut EM, Beltran J, Johnson G, Rousseau J, Marchandise X, Cotten A. Fat suppression in MR imaging: techniques and pitfalls. Radiographics 1999; 19:373-382.

11. Aydin SZ, Maksymowych WP, Bennett AN, et al. Validation of the ASAS criteria and definition of a positive MRI of the sacroiliac joint in an inception cohort of axial spondyloarthritis followed up for 8 years. Ann Rheum Dis 2012; 71:56-60. 